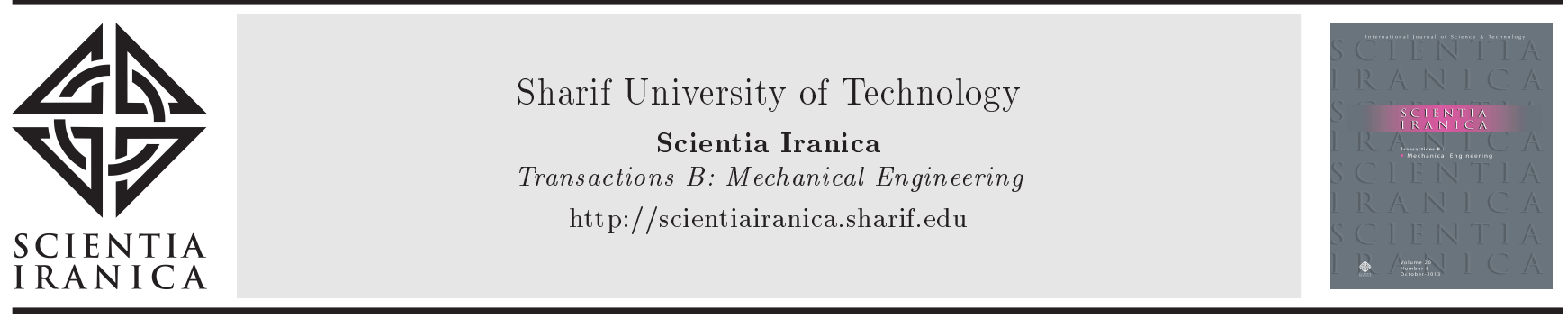

\title{
Analytical study on couple stress fluid in an inclined channel
}

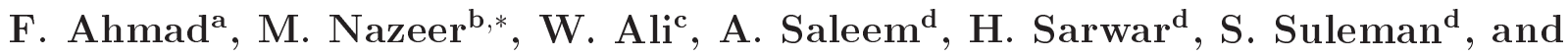 \\ Z. Abdelmalek ${ }^{\mathrm{e}, \mathrm{f}}$ \\ a. Department of Applied Science, National Textile University Faisalabad Campus 38000, Pakistan. \\ b. Department of Mathematics, Institute of Arts and Sciences, Government College University, Faisalabad, Chiniot Campus 3400 , \\ Pakistan. \\ c. Faculty of Engineering Technology University of Twente, The Netherlands. \\ d. Department of Mathematics, Riphah International University Faisalabad Campus 38000 , Pakistan. \\ e. Institute of Research and Development, Duy Tan University, Da Nang 550000, Vietnam. \\ f. Faculty of Medicine, Duy Tan University, Da Nang 550000, Vietnam.
}

Received 19 April 2020; received in revised form 20 August 2020; accepted 21 January 2021

KEYWORDS
Analytical study;
Couple stress fluid;
Constant and variable
viscosities;
Perturbation and
pseudo-spectral
methods;
Inclined channel.

\section{Introduction}

Stokes presented the theory of couple stress fluids to precisely define the motion of some special types of fluid with a microstructure, such as biological fluids, chemical suspensions, animal blood, and liquid crystals, etc.

*. Corresponding author.

E-mail address: mubbashariiui@gmail.com (M. Nazeer)

doi: $10.24200 /$ sci.2021.55579.4291

\begin{abstract}
Numerical and analytical solutions of Stokes theory of couple stress fluid under the effects of constant, space, and variable viscosity in the inclined channel are discussed in this paper. The considered couple stress fluid is described mathematically with the definition of the stress tensor. The dimensional form of the boundary value problem is transformed into dimensionless form by defining dimensionless quantities, and is then solved with the aid of the perturbation technique. The analytical expressions of velocity and temperature of all cases based on the viscosity of the couple stress fluid are presented. For the validity of the perturbation solution, the Pseudo-Spectral collocation method is employed for each case of the viscosity model including constant, space, and Vogel's models, respectively. The solution of the perturbation method and Pseudo-Spectral methods are shown together in the graphs. The effects of couple stress parameters on velocity and temperature distributions are also elaborated with physical reasoning in the results and discussion section. It is observed that the velocity and temperature of fluid escalate via the pressure gradient parameter and Brinkman number, while decelerating via the couple stress parameter.
\end{abstract}

(C) 2021 Sharif University of Technology. All rights reserved. 
tion of couple stress fluid and these equations are driven from the couple stress and anti-symmetric tensors [1]. This Stokes theory (couple stress fluid) encouraged authors, scientists, and researchers to investigate. A non-isothermal flow of couple stress fluid in an inclined channel was investigated by Farooq et al. [2]. They considered the viscosity of the fluid as a function of temperature and the perturbation method was used to find the analytical expression of velocity, temperature, volumetric flow rate and mean velocity and shear rate, etc. They concluded that all these quantities are strongly dependent on the dimensionless numbers. Eegunjobi et al. [3] analyzed the hydromagnetic flow of couple stress fluid in a vertical channel under the consideration of a porous medium. They solved the dimensionless non-linear differential equations with the help of numerical schemes, i.e. the shooting method and fourth-order Runge-Kutta Fehlberg integration. Devakar et al. [4] evaluated the exact solutions of Couette, Poiseuille and generalized Couette flows of couple stress fluid using slip boundary conditions. They found that the velocity of the fluid had a decreasing trend against the couple stress parameters. They also noted that the solution of the Couette flow for couple stress and Newtonian fluid is the same for the liming case. Further, the volumetric flow rate and fluid velocity have an inverse relation with the couple stress. Hayat et al. [5] analyzed the thermal radiation effects of rotating couple stress fluid in a variable channel. They employed the built-in NDSolve command of mathematica to obtain the solution of the problem. They used long-wavelength approximation and a small Reynolds number for simplification of field equations, and the effects of various parameters on velocity and temperature are highlighted. They noted that the velocity distribution predicts the parabolic behavior. Ellahi et al. [6] presented important research regarding the Poiseuille and Couette flow of couple stress fluid through parallel plates. They pointed out that the Hartmann number decelerated the velocity field, while viscosity parameters speed up the flow of fluid. Adesanya et al. [7] picked Adomian decomposition methods to obtain the solution of Magnetohydrodynamics (MHD) couple stress fluid under the effects of a time-periodic boundary condition in the vertical channel. Their computational results showed that skin friction at the lower wall had a decreasing trend and heat transfer rate increased versus couple stress parameters. An exact solution of couple stress fluid with the effects of size-dependent particles in an inclined channel was obtained by Adesanya et al. [8]. They observed that velocity and temperature distributions have a direct and inverse relation versus couple stress parameters, respectively. They also analyzed that the entropy and Bejan numbers decrease via couple stress parameters, but at different rates. A gravity-driven flow of couple stress fluid over a heated inclined plate under the effects of convective boundary conditions was investigated by Adesanya et al. [9]. They used Mathematica, version 8.0, software to solve the coupled boundary value problem and presented the solution in analytical form. Ashmawy [10] investigated the slip condition on the spherical particle of moving couple stress fluid. He imposed both linear slip and vanishing couple stress boundary conditions on the surface of the sphere. He obtained a simple formula for the drag force for further investigation. He concluded that with an increase in slip parameter, the drag force also increases. Ashmawy [11] modeled the couple stress fluid equations for sinusoidal corrugated pipes to investigate the effects of surface roughness on the average velocity and flow rate. The second-order regular perturbation method was employed to obtain the solution. Adesanya et al. [12] discussed the entropy analysis in couple stress fluid with an account of constant liquid properties and convective boundary conditions through an inclined channel. Hassan [13] used the Adomian decomposition method to solve the nonlinear equations of the hydromagnetic flow of couple stress fluid through porous walls. They also discussed the entropy analysis in his study. Zeeshan et al. [14] discussed properties of gravity and magnetic field influences on couple stress fluid with the addition of nanoparticles. An important analysis of the properties of variable viscosity for the flow of non-Newtonian fluid was presented by Ellahi [15]. He calculated the velocity and temperature of the fluid for the Reynolds and Vogel's viscosity models. Jabeen et al. [16] highlighted the effects of nonlinear thermal radiation and joule heating on the flow of Tangent Hyperbolic fluid. They observed that the concentration of fluid gets the higher values via moderate values of the activation energy parameter. Bayat and Rahimi [17] used a simple algorithm employing the finite difference method to solve the Navier-Stokes and heat equations of the stagnation point flow over a vertical cylinder.

From the above-cited investigations, it is observed that no investigation has yet been undertaken into the Stokes's theory (couple stress fluid) under the consideration of a space-dependent viscosity model, Vogel's viscosity model and constant viscosity in an inclined channel. In view of this, the objective of the investigation in this paper is to examine the heat and mass transfer of couple stress fluid taking into account the constant and variable viscosity properties of couple stress fluid in an inclined channel. Further, analytical solutions of velocity and temperature will be obtained through the perturbation technique. For the validity of the perturbation technique, an eminent Pseudo-Spectral collocation method will be employed, due to its efficiency and fast convergence. Moreover, this method is applicable to obtain the solutions of 
complex coupled initial and boundary value problems. The effects of couple stress parameters on velocity and temperature distributions are also illustrated with physical reasoning. It is observed that the velocity and temperature of fluid escalate via the pressure gradient parameter and Brickman number, while decelerating via the couple stress parameter.

\section{Mathematical formulation of couple stress fluid}

Consider the couple stress fluid in an inclined channel with a variable and constant viscosity property. The channel is inclined with an angle of inclination $\alpha$ and the distance between the lower and top walls is $2 d$. It is assumed that the flow is flowing along the $x$-direction as shown in Figure 1.

The basic equations governing the flow of an incompressible couple stress fluid are given by $[2,6,14]$ :

$$
\begin{aligned}
& \operatorname{div} \overline{\mathbf{V}}=0 \\
& \begin{aligned}
\rho_{1} \frac{D \overline{\mathbf{V}}}{D t}= & \operatorname{div} \overline{\mathbf{T}}-\bar{\mu}(\nabla \times \nabla \times \overline{\mathbf{V}}) \\
& \quad-\eta_{1}(\nabla \times \nabla \times \nabla \times \nabla \times \overline{\mathbf{V}})+\rho_{1} \mathbf{f}_{0}, \\
\rho_{1} c_{p} \frac{D \bar{\theta}}{D t}= & k_{1} \nabla^{2} \bar{\theta}+\operatorname{tra}\left(\overline{\boldsymbol{\tau}} \cdot L_{0}\right)+\eta_{1}\left(\nabla^{2} \overline{\mathbf{V}}\right)^{2}
\end{aligned}
\end{aligned}
$$

where $\overline{\mathbf{V}}$ is the velocity vector, $\rho_{1}$ the constant density; $\mathbf{f}_{0}$ the body force per unit mass; $\overline{\mathbf{T}}$ the Cauchy stress tensor; $\bar{\theta}$ the temperature; $k_{1}$ the thermal conductivity; $c_{p}$ the specific heat; $\mathbf{L}_{0}$ the gradient of the velocity vector; and $\eta_{1}$ the couple stress parameter.

The Cauchy stress tensor $\overline{\mathbf{T}}$ can be defined as:

$$
\overline{\mathbf{T}}=-p_{0} \mathbf{I}_{1}+\overline{\boldsymbol{\tau}}, \quad \overline{\boldsymbol{\tau}}=\bar{\mu} \mathbf{A}_{0},
$$

where:

$p_{0} \quad$ the dynamic pressure,

$\mathbf{I}_{1} \quad$ the unit tensor,

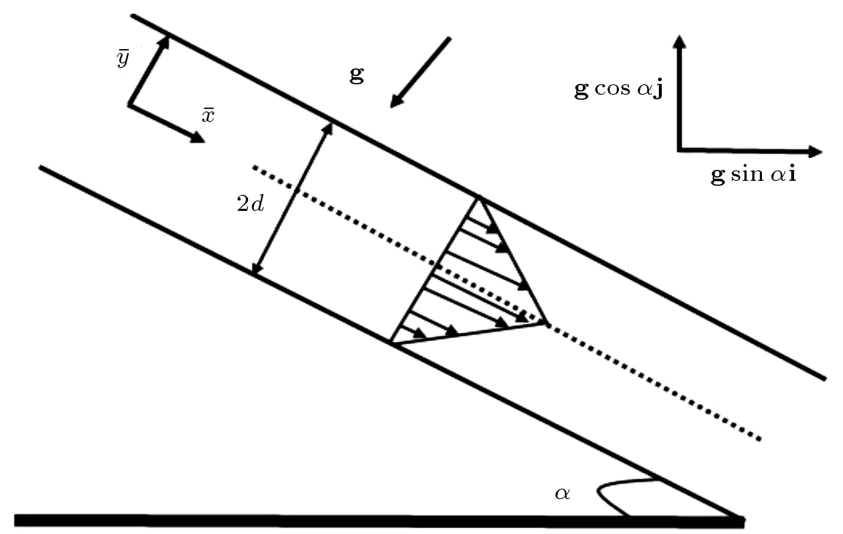

Figure 1. Geometry of the given problem. $\bar{\mu} \quad$ the coefficient of viscosity,

$\mathbf{A}_{0} \quad$ the first Rivlin-Ericksen tensor.

In this study, the following has been chosen:

$$
\mathbf{V}=(\bar{u}(\bar{y}), 0,0) \quad \text { and } \quad \bar{\theta}=\bar{\theta}(\bar{y}) .
$$

Using this selection, Eqs. (1)-(3) can be written as:

$$
\begin{aligned}
& \frac{\partial \bar{u}}{\partial \bar{x}}=0, \\
& \frac{-\partial p_{0}}{\partial \bar{z}}=0 \\
& \frac{-\partial p_{0}}{\partial \bar{y}} \rho_{1} \bar{g} \cos \alpha=0 \\
& \eta_{1} \frac{d^{4} \bar{u}}{d \bar{y}^{4}}-\bar{\mu} \frac{d^{2} \bar{u}}{d \bar{y}^{2}}-\frac{d \bar{\mu}}{d \bar{y}} \cdot \frac{d \bar{u}}{d \bar{y}}+\frac{\partial p_{0}}{\partial \bar{x}}-\rho_{1} \bar{g} \sin \alpha=0, \\
& \frac{d^{2} \bar{\theta}}{d \bar{y}^{2}}+\frac{\bar{\mu}}{k_{1}}\left(\frac{d \bar{u}}{d \bar{y}}\right)^{2}+\frac{\eta_{1}}{k_{1}}\left(\frac{d^{2} \bar{u}}{d \bar{y}^{2}}\right)^{2}=0 .
\end{aligned}
$$

Eqs. (9) and (10) are known as momentum equations or equations of motion and heat equation or energy equations, respectively. The boundary conditions of Eqs. (9) and (10) are:

$$
\begin{array}{lll}
\bar{u}(-d)=0, & \bar{u}(d)=0, & \frac{d^{2} \bar{u}}{d \bar{y}^{2}}(-d)=0, \\
\frac{d^{2} \bar{u}}{d \bar{y}^{2}}(d)=0, & \bar{\theta}(-d)=\bar{\theta}_{0}, & \bar{\theta}(d)=\bar{\theta}_{1} .
\end{array}
$$

For the achievement of the dimensionless form of Eqs. (9)-(11), the dimensionless quantities are introduced in the following form:

$$
\mu=\frac{\bar{\mu}}{\mu_{0}}, \quad u_{1}=\frac{\bar{u}}{u_{0}}, \quad y=\frac{\bar{y}}{d}, \quad \theta=\frac{\bar{\theta}-\bar{\theta}_{0}}{\bar{\theta}_{1}-\bar{\theta}_{0}} .
$$

With the involvement of the above quantities, the dimensionless form of Eqs. (9)-(11) are presented as:

$$
\begin{aligned}
& \frac{d^{4} u}{d y^{4}}-B_{0}^{2} \mu \frac{d^{2} u}{d y^{2}}-B_{0}^{2} \frac{d \mu}{d y} \frac{d u}{d y}-G=0, \\
& \frac{d^{2} \theta}{d y^{2}}+\operatorname{Br}^{\Theta} \mu\left(\frac{d u}{d y}\right)^{2}+\frac{\operatorname{Br}^{\Theta}}{B_{0}^{2}}\left(\frac{d^{2} u}{d y^{2}}\right)^{2}=0, \\
& u( \pm 1)=0, \quad \frac{d^{2} u}{d y^{2}}( \pm 1)=0, \quad \theta(-1)=0, \\
& \theta(1)=1,
\end{aligned}
$$

where:

$$
\begin{aligned}
& \operatorname{Br}^{\Theta}=\frac{\mu_{0} u_{1}^{2}}{k_{1}\left(\theta_{m}-\theta_{w}\right)}, \quad B_{0}^{2}=\frac{\mu_{0} d^{2}}{\eta_{1}}, \\
& G=\frac{-B_{0} d^{5}}{\mu_{0} u_{1}} \frac{\partial p_{0}}{\partial x}+\frac{\rho_{1} \bar{g} d^{4}}{\eta_{1} u_{1}} \sin \alpha .
\end{aligned}
$$




\section{Viscosity models}

Three different viscosity models are chosen, in which one is constant viscosity and the other two are variable viscosity models, i.e.:

\section{(i) Constant viscosity case $(\mu=1)$}

In the case of the constant viscosity model, Eqs. (13)(15) take the following form:

$$
\begin{aligned}
& \frac{d^{4} u}{d y^{4}}-B_{0}^{2} \frac{d^{2} u}{d y^{2}}-G=0 \\
& \frac{d^{2} \theta}{d y^{2}}+\operatorname{Br}^{\Theta}\left(\frac{d u}{d y}\right)^{2}+\frac{\operatorname{Br}^{\Theta}}{B_{0}^{2}}\left(\frac{d^{2} u}{d y^{2}}\right)^{2}=0
\end{aligned}
$$

An exact solution of Eqs. (16) and (17) is defined by:

$$
\begin{aligned}
u= & a_{0}+a_{1}\left(1-y^{2}\right)+a_{2} \cosh \left[B_{0} y\right], \\
\theta= & b_{0}+b_{1} y+b_{2} y^{2}+b_{3} y^{3}+b_{4} \cosh \left[B_{0} y\right] \\
& +b_{5} \cosh \left[2 B_{0} y\right]+b_{6} \sinh \left[B_{0} y\right] .
\end{aligned}
$$

\section{(ii) Space-dependent viscosity model $(\mu=y)$}

The case of the space-dependent viscosity model is chosen based on important studies presented by Ellahi and Riaz [18], Hayat et al. [19] and Nazeer et al. [20]. In these studies, the authors have chosen viscosity as a linear function of space coordinate (i.e., $\mu=r$ ). Motivated by the concept of these studies, in this paper, the authors' have taken $\mu=y$. Given this assumption, Eqs. (13) and (14) are given by:

$$
\begin{aligned}
& \frac{d^{4} u}{d y^{4}}-B_{0}^{2} y \frac{d^{2} u}{d y^{2}}-B_{0}^{2} \frac{d u}{d y}-G=0, \\
& \frac{d^{2} \theta}{d y^{2}}+\operatorname{Br}^{\Theta} y\left(\frac{d u}{d y}\right)^{2}+\frac{\operatorname{Br}^{\Theta}}{B_{0}^{2}}\left(\frac{d^{2} u}{d y^{2}}\right)^{2}=0 .
\end{aligned}
$$

To solve Eqs. (20) and (21) under the boundary condition given in Eq. (15), the method of regular perturbation has been used. The two-term regular perturbation expansion of velocity and temperature are defined by:

$$
u \approx u_{0}+\varepsilon u_{1}+O\left(\varepsilon^{2}\right)
$$

and:

$$
\theta \approx \theta_{0}+\varepsilon \theta_{1}+O\left(\varepsilon^{2}\right)
$$

The system of velocity for each order of $\varepsilon$ is:

$$
\begin{aligned}
O\left(\varepsilon^{0}\right): \quad \frac{d^{4} u_{0}}{d y^{4}}-B_{0}^{2} y \frac{d^{2} u_{0}}{d y^{2}}-B_{0}^{2} \frac{d u_{1}}{d y}-G & =0, \\
u_{0}( \pm 1)=0, \quad \frac{d^{2} u_{0}}{d y^{2}}( \pm 1) & =0 \\
O\left(\varepsilon^{1}\right): \quad \frac{d^{4} u_{1}}{d y^{4}}-B_{0}^{2} y \frac{d^{2} u_{1}}{d y^{2}}-B_{0}^{2} \frac{d u_{1}}{d y} & =0 \\
u_{1}( \pm 1) & =0, \quad \frac{d^{2} u_{1}}{d y^{2}}( \pm 1)=0 .
\end{aligned}
$$

The system of temperature for each order of $\varepsilon$ is:

$$
\begin{gathered}
O\left(\varepsilon^{0}\right): \quad \frac{d^{4} \theta_{0}}{d y^{4}}+\operatorname{Br}^{\Theta} y \frac{d^{2} u_{0}}{d y^{2}}+\frac{\mathrm{Br}^{\Theta}}{B_{0}^{2}}\left(\frac{d^{2} u_{0}}{d y^{2}}\right)^{2}=0 \\
\theta_{0}(-1)=0, \quad \theta_{0}(1)=1 \\
O\left(\varepsilon^{1}\right): \quad \frac{d^{4} \theta_{1}}{d y^{4}}+2 \operatorname{Br}^{\Theta} y\left(\frac{d u_{0}}{d y}\right)\left(\frac{d u_{1}}{d y}\right) \\
+2 \frac{\operatorname{Br}^{\Theta}}{B_{0}^{2}}\left(\frac{d^{2} u_{0}}{d y^{2}}\right)\left(\frac{d^{2} u_{1}}{d y^{2}}\right)=0 \\
\theta_{1}( \pm)=0 .
\end{gathered}
$$

The solution of each order of $\varepsilon$ for the velocity profile is:

$$
\begin{aligned}
& u_{0}=a_{3}+a_{4} y^{2}+a_{5} y^{4}, \\
& u_{1}=a_{6} y+a_{7} y^{3}+a_{8} y^{5}+a_{9} y^{7} .
\end{aligned}
$$

Based on leading-order solutions, the determining equation for the correction term gives:

$$
\begin{aligned}
& \theta_{0}=b_{7}+b_{8} y+b_{9} y^{2}+b_{10} y^{4}+b_{11} y^{7}, \\
& \theta_{1}=b_{12} y+b_{13} y^{3}+b_{14} y^{5}+b_{15} y^{7}+b_{16} y^{9} .
\end{aligned}
$$

Combining the leading-order and first-order solutions, one has:

$$
\begin{aligned}
u= & a_{3}+a_{4} y^{2}+a_{5} y^{4}+a_{6} y+a_{7} y^{3}+a_{8} y^{5}+a_{9} y^{7} \\
\theta= & b_{7}+b_{8} y+b_{9} y^{2}+b_{10} y^{4}+b_{11} y^{7}+b_{12} y+b_{13} y^{3} \\
& +b_{14} y^{5}+b_{15} y^{7}+b_{16} y^{9}
\end{aligned}
$$

\section{(iii) Vogel's model (temperature dependent)}

In this case, the viscosity of the fluid is a function of temperature, i.e. the viscosity is directly dependent on the temperature of the fluid. In this, the following relation for the representation of the Vogel's viscosity model is defined as [21-24]:

Let:

$$
\mu=e^{\left(\frac{x_{0}}{y_{0}+\theta}-\theta_{w}\right)},
$$

or: 


$$
\mu=e^{\left(\frac{x_{0}}{y_{0}+\theta}-\theta_{w}\right)}=e^{\left(\frac{x_{0}}{y_{0}}-\theta_{w}\right)} \cdot\left(1-\frac{x_{0}}{y_{0}^{2}} \theta\right) .
$$

Using the above values, Eqs. (13) and (14) become:

$$
\begin{gathered}
\frac{d^{4} u}{d y^{4}}-B_{0}^{2} e^{\left(\frac{x_{0}}{y_{0}}-\theta_{w}\right)} \cdot\left(1-\frac{x_{0}}{y_{0}^{2}} \theta\right) \frac{d^{2} u}{d y^{2}} \\
+B_{0}^{2} \frac{x_{0}}{y_{0}^{2}} e^{\left(\frac{x_{0}}{y_{0}}-\theta_{w}\right)} \frac{d u}{d y} \cdot \frac{d \theta}{d y}-G=0, \\
\frac{d^{2} \theta}{d y^{2}}+B_{r}^{\Theta} e^{\left(\frac{x_{0}}{y_{0}}-\theta_{w}\right)} \cdot\left(1-\frac{x_{0}}{y_{0}^{2}} \theta\right)\left(\frac{d u}{d y}\right)^{2} \\
+\frac{B_{r}^{\Theta}}{B_{0}^{2}}\left(\frac{d^{2} u}{d y^{2}}\right)^{2}=0 .
\end{gathered}
$$

In view of the perturbation technique;

$$
\begin{aligned}
& O\left(\varepsilon^{0}\right): \quad \frac{d^{4} u_{0}}{d y^{4}}- B_{0}^{2} e^{\left(\frac{x_{0}}{y_{0}}-\theta_{w}\right)} \cdot\left(1-\frac{x_{0}}{y_{0}^{2}} \theta\right) \\
& \cdot \frac{d^{2} u_{0}}{d y^{2}}+B_{0}^{2} e^{\left(\frac{x_{0}}{y_{0}}-\theta_{w}\right)} \frac{x_{0}}{y_{0}^{2}} \\
& \cdot \frac{d \theta}{d y}\left(\frac{d u_{0}}{d y}\right)-G=0, \\
& O\left(\varepsilon^{1}\right): \quad \frac{d^{4} u_{1}}{d y^{4}}-B_{0}^{2} e^{\left(\frac{x_{0}}{y_{0}}-\theta_{w}\right)} \cdot\left(1-\frac{x_{0}}{y_{0}^{2}} \theta\right) \\
& \cdot \frac{d^{2} u_{1}}{d y^{2}}+B_{0}^{2} e^{\left(\frac{x_{0}}{y_{0}}-\theta_{w}\right)} \frac{x_{0}}{y_{0}^{2}} \\
& \cdot \frac{d \theta}{d y}\left(\frac{d u_{1}}{d y}\right)-G=0 .
\end{aligned}
$$

The system of temperature for each order is:

$$
\begin{array}{r}
O\left(\varepsilon^{0}\right): \quad \frac{d^{2} \theta_{0}}{d y^{2}}+B_{r}^{\Theta} e^{\left(\frac{x_{0}}{y_{0}}-\theta_{w}\right)} \cdot\left(1-\frac{x_{0}}{y_{0}^{2}} \theta\right) \\
\cdot\left(\frac{d u_{0}}{d y}\right)^{2}+\frac{B_{r}^{\Theta}}{B_{0}^{2}}\left(\frac{d^{2} u_{0}}{d y^{2}}\right)^{2}=0 \\
O\left(\varepsilon^{1}\right): \quad \frac{d^{2} \theta_{1}}{d y^{2}}+2 B_{r}^{\Theta} e^{\left(\frac{x_{0}}{y_{0}}-\theta_{w}\right)} \\
\cdot\left(1-\frac{x_{0}}{y_{0}^{2}} \theta\right)\left(\frac{d u_{0}}{d y}\right)\left(\frac{d u_{1}}{d y}\right) \\
+2 \frac{B_{r}^{\Theta}}{B_{0}^{2}}\left(\frac{d^{2} u_{0}}{d y^{2}}\right)\left(\frac{d^{2} u_{1}}{d y^{2}}\right)=0 .
\end{array}
$$

The solution of each order of $\varepsilon$ for the velocity profile is:

$$
u_{0}=a_{10}\left(5-6 y^{2}+y^{4}\right),
$$

$$
\begin{aligned}
u_{1}= & B_{0}^{2}\left(a_{18}+a_{19} y+a_{20} y^{2}+a_{21} y^{3}+a_{11} y^{4}+a_{12} y^{5}\right. \\
& \left.+a_{13} y^{6}+a_{14} y^{7}+a_{15} y^{8}+a_{16} y^{10}+a_{17} y^{12}\right) .
\end{aligned}
$$

The solution for each order of $\varepsilon$ for the temperature profile is:

$$
\begin{aligned}
\theta_{0}= & b_{18}+b_{19} y+b_{17}\left(15 y^{2}-5 y^{4}+y^{6}\right) \\
\theta_{1}= & B_{r}^{\Theta}\left(b_{31}+b_{32} y+b_{20} y^{2}+b_{21} y^{3}+b_{22} y^{4}\right. \\
& +b_{23} y^{5}+b_{24} y^{6}+b_{25} y^{7}+b_{26} y^{8}+b_{27} y^{9} \\
& \left.+b_{28} y^{10}+b_{29} y^{12}+b_{30} y^{14}\right) .
\end{aligned}
$$

Combining the leading-order and first-order solutions, one has:

$$
\begin{aligned}
u= & a_{10}\left(5-6 y^{2}+y^{4}\right)+B_{0}^{2}\left(a_{18}+a_{19} y+a_{20} y^{2}\right. \\
& +a_{21} y^{3}+a_{11} y^{4}+a_{12} y^{5}+a_{13} y^{6}+a_{14} y^{7} \\
& \left.+a_{15} y^{8}+a_{16} y^{10}+a_{17} y^{12}\right), \\
\theta= & b_{18}+b_{19} y+b_{17}\left(15 y^{2}-5 y^{4}+y^{6}\right) \\
& +B_{r}^{\Theta}\left(b_{31}+b_{32} y+b_{20} y^{2}+b_{21} y^{3}+b_{22} y^{4}\right. \\
& +b_{23} y^{5}+b_{24} y^{6}+b_{25} y^{7}+b_{26} y^{8}+b_{27} y^{9} \\
& \left.+b_{28} y^{10}+b_{29} y^{12}+b_{30} y^{14}\right) .
\end{aligned}
$$

\section{Pseudo-spectral collocation method}

The coupled system of nonlinear equations is discretized using a pseudo-spectral collocation method [25]. It is well-known that the pseudo-spectral collocation method offers high accuracy in the approximation of derivatives. Eqs. (13) and (14) are nonlinear and represent second-order boundary value problems. The domain of the problems here is $[0,1]$. Usually, the matrix operators in the pseudo-spectral collocation method to approximate the derivatives are defined over the domain $[-1,1]$, and there is a transformation $\Gamma$ from $[-1,1]$ to $[0,1]$ i.e. $\Gamma=[-1,1] \rightarrow[0,1]$ :

$$
\Gamma(r)=0.5(r+1) \text {. }
$$

The domain of the problem presented here $[0,1]$ is discretized and $n$-grid points are introduced:

$$
\eta_{i}=(i-1) h, \quad \text { for } \quad i=1,2,3, \cdots, n .
$$

Here, $h=1 /(n-1)$. If the differentiation pseudospectral collocation matrix is denoted by $\overline{\mathbf{D}}$, then, the matrix operator $\mathbf{D}$ that approximates the first-order derivative in this problem is:

$\mathbf{D}=2 \overline{\mathbf{D}}$. 
The beauty of the pseudo-spectral collocation method is that higher-order derivatives can be computed by computing the power of $\mathbf{D}$, i.e. the $i$ th order derivative can be approximated by $\mathbf{D}^{i}$. The discretized form of the boundary value problem is:

$$
\begin{aligned}
& D^{4} u-B_{0}^{2} \mu \odot D^{2} u-B_{0}^{2} D \mu \odot D u-G=0, \\
& D^{2} \theta+B_{r}^{\Theta}\left(\mu \odot(D u)^{2}-\frac{1}{B_{0}^{2}}\left(D^{2} u\right)^{2}\right)=0,
\end{aligned}
$$

where:

$$
u=\left[\begin{array}{c}
u_{1} \\
u_{2} \\
\vdots \\
u_{n}
\end{array}\right]_{n \times n}, \quad \theta=\left[\begin{array}{c}
\theta_{1} \\
\theta_{2} \\
\vdots \\
\theta_{n}
\end{array}\right]_{n \times n}, \quad 1=\left[\begin{array}{c}
1 \\
1 \\
\vdots \\
1
\end{array}\right]_{n \times n} .
$$

$u_{i}=u\left(\eta_{i}\right)$ and $\theta_{i}=\theta\left(\eta_{i}\right)$. The system of nonlinear equations in Eq. (40) can be written as:

$$
\begin{aligned}
\mathbf{F} & =\left[\begin{array}{l}
D^{4} u-B_{0}^{2} \mu \odot D^{2} u-B_{0}^{2} D \mu \odot D u-G \\
D^{2} \theta+B_{r}^{\Theta}\left(\mu \odot(D u)^{2}-\frac{1}{B_{0}^{2}}\left(D^{2} u\right)^{2}\right)
\end{array}\right] \\
& =\left[\begin{array}{l}
0 \\
0
\end{array}\right] .
\end{aligned}
$$

The boundary conditions can be imposed as:

$$
\begin{aligned}
& F(1)=u(1), \quad F(2)=D(1,1: n) u, \\
& F(n-1)=(n, 1: n) u, \quad F(n)=u(n), \\
& F(n+1)=\theta(1), \quad F(2 n-1)=\theta(n)-1,
\end{aligned}
$$

where $u(1)=0, D(1,1: n) u=0,(n, 1: n) u=0$ and $u(n)=0, \theta(1)=0$, and $\theta(n)=1$ describes the boundary conditions. The system of nonlinear Eqs. (41) (after the adjustment of boundary conditions) can be solved by using the Newton method. The Newton method [26-32] is used in the following form:

$$
z_{k+1}=z_{k}-\left(\mathbf{F}^{\prime}\left(z_{k}\right)\right)^{-1} \mathbf{F}\left(z_{k}\right), \quad k=0,1,2, \cdots,
$$

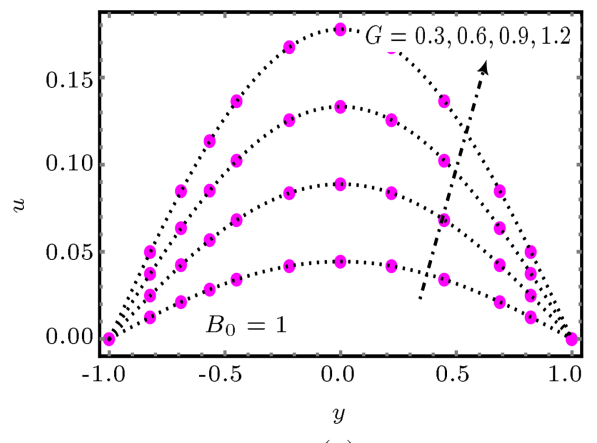

(a) where $z=[u, \theta]^{T}$, and $z_{0}=0$. In all simulations, 0 is taken as an initial guess, and on average, numerical accuracy is obtained in the solution of a system of nonlinear equations.

\section{Results and discussion}

In the present paper, the unidirectional flow of nonNewtonian fluid (couple stress) between two inclined plates is discussed. The inclined plates are set at angle $\alpha$ from the horizontal axis. The perturbation technique is chosen to obtain analytical expressions for flow (velocity) and temperature fields. The effects of Brickman number $B_{r}^{\Theta}$, pressure gradient parameter $G$, couple stress parameter $B_{0}$, the temperature of the wall parameter $\theta_{w}$ and Vogel's model parameter $y_{0}$, on the velocity and temperature of the couple stress fluid are captured through graphs. The velocity and temperature graphs for each case, i.e. Constant viscosity case, Space-dependent viscosity case, and Vogel's model, are prepared separately. In all figures, dotted lines are selected for identification of exact solutions, and solid circles are chosen to show the description of the numerical solutions, which is obtained by the eminent pseudospectral collocation method. The ranges of parameters in the present simulations are selected as $1 \leq B_{0} \leq 4$, $1 \leq B_{r}^{\Theta} \leq 1.6,0.1 \leq G \leq 8,0 \leq \theta_{w} \leq 1,0.2 \leq y_{0} \leq 1$, and $x_{0}=1$. Figures 2 and 3 illustrate the effects of pertinent parameters on velocity and temperature profiles for the uniform viscosity model. It is noticed that the velocity of the couple stress fluid increases versus pressure gradient parameter, while the reverse trend is highlighted against couple stress parameter $B_{0}$. Since, by increasing the values of the pressure gradient parameter, the fluid covers more area in the vicinity of inclined plates, and, hence, the velocity speeds up, the couple stress parameter increases the viscosity within the fluid, which declines the flow of fluid. Further, it is observed that the nature of the velocity against both parameters is the parabolic type and the velocity of the couple stress fluid attains maximum values in the center of the channel. The temperature profiles of the

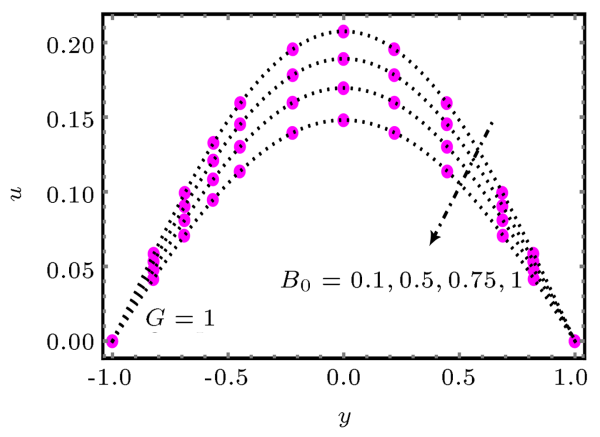

(b)

Figure 2. Effects of physical parameters on the velocity of constant viscosity. 


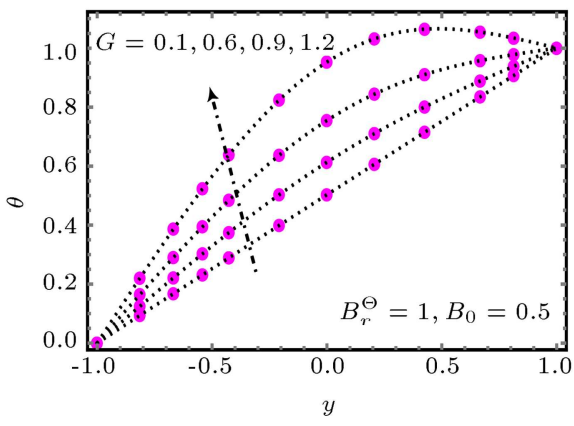

(a)

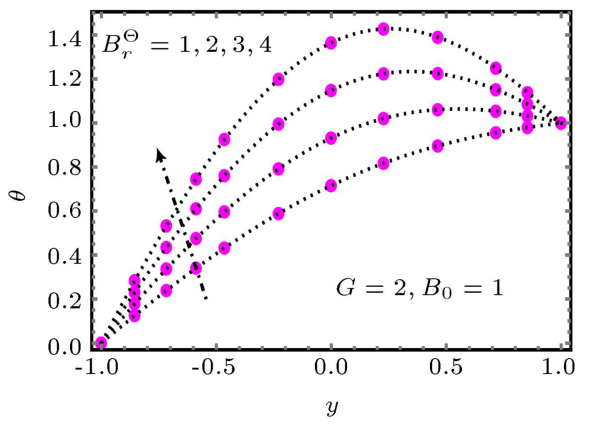

(b)

Figure 3. Effects of physical parameters on the temperature of the constant viscosity.

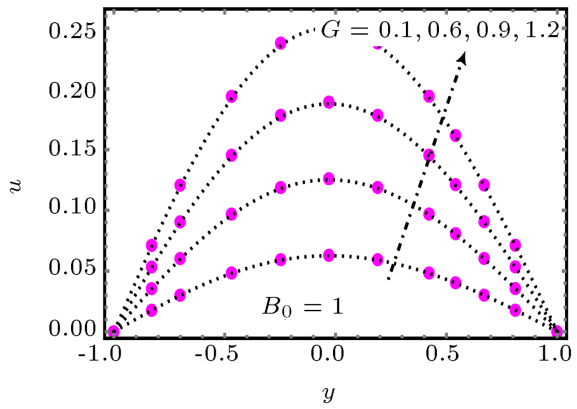

(a)

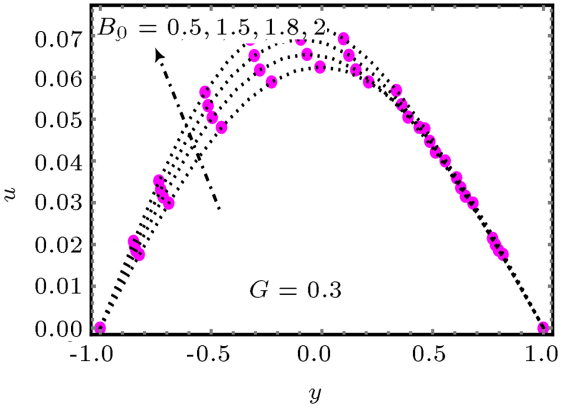

(b)

Figure 4. Effects of physical parameters on the velocity of the space-dependent viscosity.

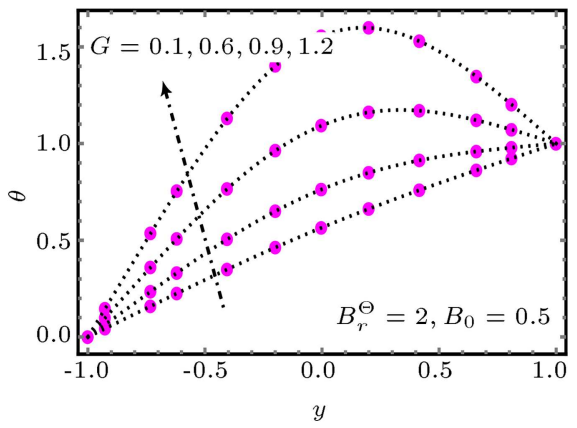

(a)

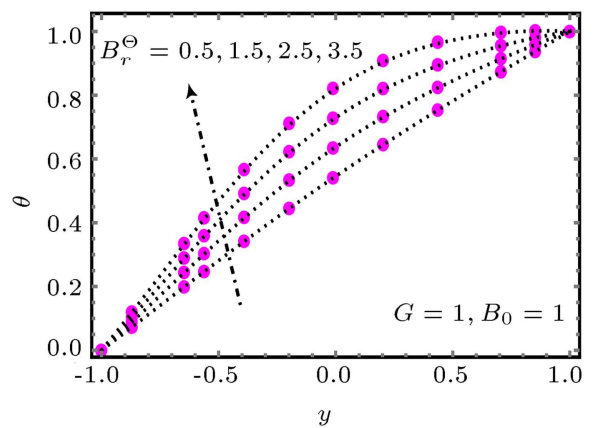

(b)

Figure 5. Effects of physical parameters on the temperature of the space-dependent viscosity.

couple stress fluid via pressure gradient parameter and Brickman number are shown in Figure 3. It is perceived that the temperature of the fluid increases for both the pressure gradient parameter and Brickman number. It is reasoned that the pressure gradient parameter escalates the kinetic energy of the fluid which means more heat is produced within the fluid and, hence, an increase in the temperature of the fluid is observed in this situation. When the values of Brinkman number increase, viscous dissipation effects are produced within the fluid which enhances heat generation. This is the reason behind acceleration in the temperature field.

The plots for the space-dependent viscosity model are illustrated in Figures 4 and 5. The effects of pertinent involved parameters on velocity are presented in Figure 4 and on the temperature in Figure 5. The nature of velocity is the same parabolic type as discussed in the case of the uniform viscosity model against the pressure gradient parameter $G$. It is observed that the velocity of the couple stress fluid is maximum for this case, compared to the variable viscosity case. Figure 4(b) depicts that the velocity of the fluid increases near the left boundary and decreases near the right boundary, when the values of the couple stress parameter $B_{0}$ are increased. Figure 5 interprets that the temperature of fluid accelerates against the pressure gradient parameter and Brickman number. These effects are similar to the above case (constant viscosity model).

Figures 6 and 7 are presented to capture the 


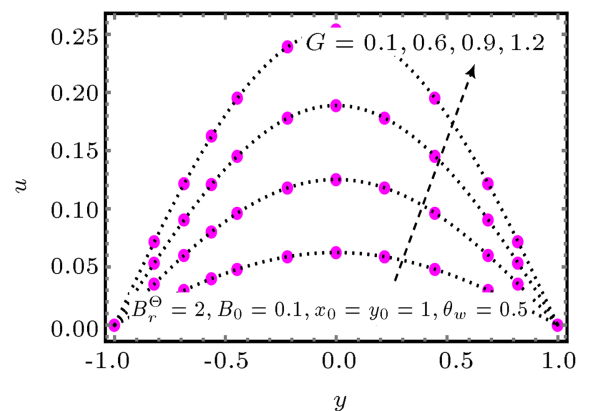

(a)

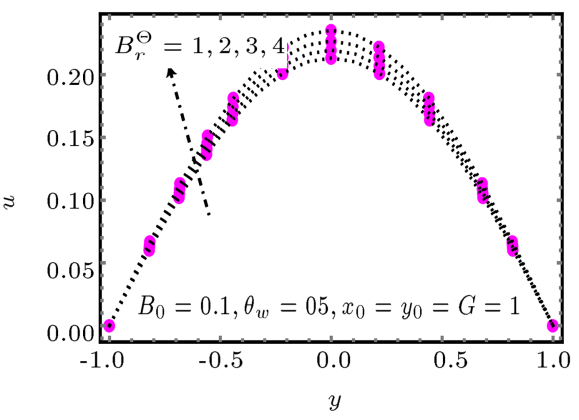

(c)

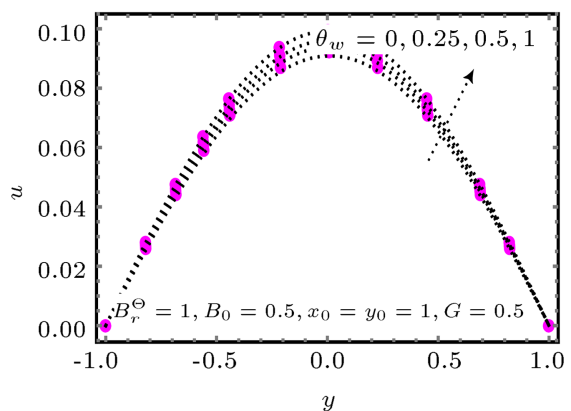

(b)

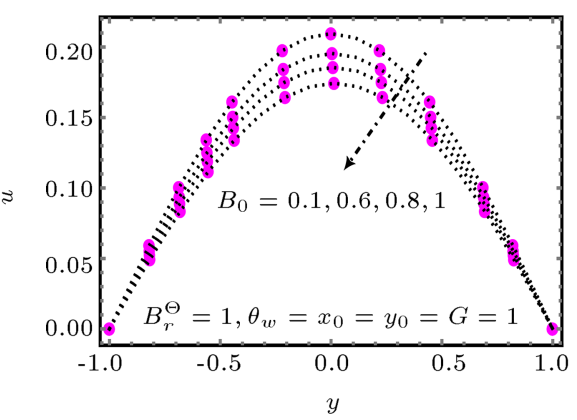

(d)

Figure 6. Effects of physical parameters on the velocity of Vogel's viscosity.

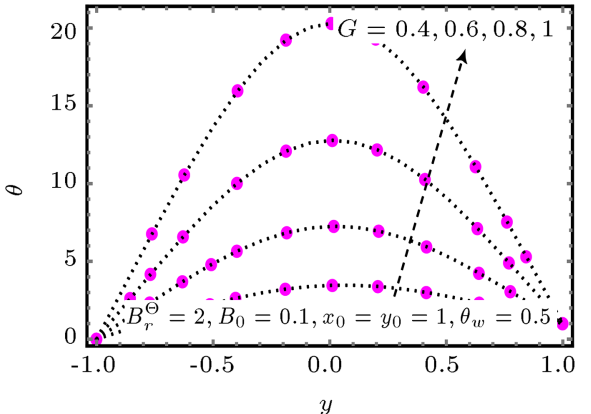

(a)

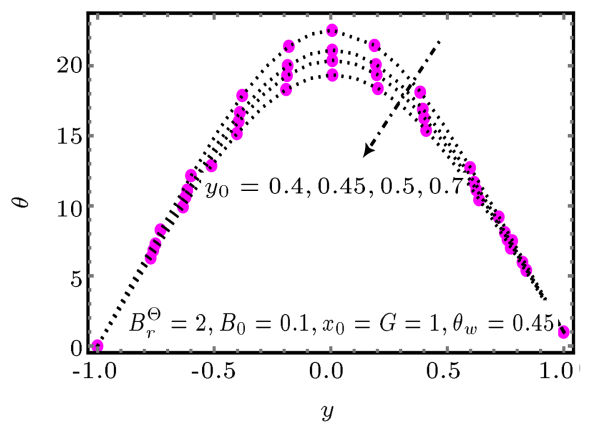

(c)

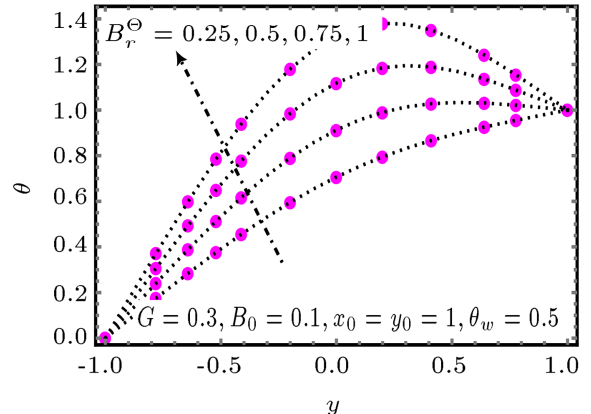

(b)

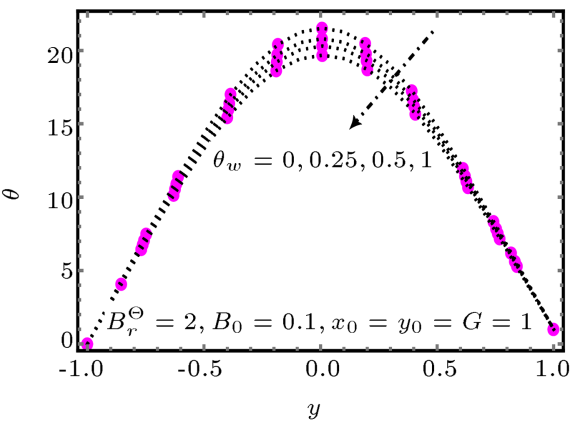

(d)

Figure 7. Effects of pressure gradient parameter, Brinkman number, Veogel's viscosity parameter and wall temperature on temperature profile.

effects of the non-dimensional parameter on velocity and temperature for the third case of the viscosity model, i.e. Vogel's model. It is noted that the velocity of the couple stress fluid increases via the Brickman number, pressure gradient parameter, and temperature of wall parameter, while opposite results are noted via the couple stress parameter (see Figure 6). The distribution of the temperature profiles in the case of Vogel's model via non-dimensional parameters is presented in Figure 7. From this figure, it is perceived that the non- 
dimensional temperature of the fluid is an increasing function of pressure parameter and Brickman number and a decreasing function of Vogel's model parameter and temperature of the wall parameter. These effects are similar to the above two cases.

\section{Conclusions}

The properties of heat and mass transfer of couple stress fluids between two parallel inclined plates are discussed in this work. The velocity and temperature solutions for the constant and variable viscosity (spacedependent and Vogel's models) of the couple stress fluid are obtained. For analytical solutions, the authors used the perturbation method and obtained the analytical expressions of velocity and temperature of each case of viscosity model. A pseudo-spectral collocation numerical scheme is also used for the validity of the perturbation solutions. The effects of the physical parameters of the couple stress fluid on velocity and temperature profiles are highlighted with the help of graphs. It is noticed that the temperature and velocity on the plates are strongly dependent on the non-dimensional parameters, namely Brickman number $B_{r}^{\Theta}$, pressure gradient parameter $G$, couple stress parameter $B_{0}$ and temperature of wall parameter $\theta_{w}$. It is observed that the velocity shows an increasing behavior versus the pressure gradient parameter, Brickman number, and Vogel's model parameter, while an opposite behavior is noted versus a couple of stress parameters. The temperature of the fluid increases via the pressure parameter and Brickman number, while the opposite behavior is noted via the Vogel model parameter and the wall temperature of the wall parameter.

\section{Nomenclature}

$B_{0} \quad$ Couple stress parameter

$\mathbf{f}_{0} \quad$ Body force per unit mass

$G \quad$ Pressure gradient parameter

$\alpha \quad$ Inclination angle

$\mathbf{L}_{0} \quad$ Gradient of $\overline{\mathbf{V}}$

$\bar{\mu} \quad$ Viscosity of fluid

$\mathbf{T} \quad$ Cauchy stress tensor

d Distance between pltes

$\varepsilon \quad$ Small perturbation parameter

$B_{r}^{\Theta} \quad$ Brinkman number

$\mathbf{A}_{0} \quad$ First Rivilin-Erickson tensor

$\bar{\tau} \quad$ Extra stress tensor

$\theta_{w} \quad$ The temperature of the wall

$\rho_{1} \quad$ Constant density

$\overline{\mathbf{V}} \quad$ Velocity vector $\bar{u}$

D

$k_{1} \quad$ Thermal conductivity

$c_{p} \quad$ Specific heat

$\mathbf{I}_{1} \quad$ Unit tensor

$\bar{\theta}_{0} \quad$ The temperature of the lower wall

$\bar{\theta}_{1} \quad$ The temperature of the upper wall

$\theta \quad$ Dimensionless temperature

$u \quad$ Dimensionless form of the velocity component

$u_{0} \quad$ Arbitrary reference velocity

$y \quad$ Dimensionless form of space coordinate

\section{References}

1. Stokes, V.K., Theories of Fluids with Microstructure, Springer, New York (1984).

2. Farooq, M., Rahim, M.T., Islam, S., et al. "Steady poiseuille flow and heat transfer of couple stress fluids between two parallel inclined plates with variable viscosity", J. Assoc. Arab. Univ. Basic Appl. Sci., 14, pp. $9-18$ (2013).

3. Eegunjobi, A.S. and Makinde, O.D. "Irreversibility analysis of hydromagnetic flow of couple stress fluid with radiative heat in a channel filled with a porous medium", Results Phy., 7, pp. 459-469 (2017).

4. Devakar, M., Sreenivasu, D., and Shankar, B. "Analytical solutions of couple stress fluid flows with slip boundary conditions", Alex Eng. J., 53, pp. 723-730 (2014).

5. Hayat, T., Zahir, H., Alsaedi, A., et al. "Peristaltic flow of rotating couple stress fluid in a non-uniform channel", Results Phys., 7, pp. 2865-2873 (2017).

6. Ellahi, R., Zeeshan, A., Hussain, F., et al. "Two-phase couette flow of couple stress fluid with temperature dependent viscosity thermally affected by magnetized moving surface", Symmetry, 11, pp. 647-660 (2019).

7. Adesanya, S.O., Makhalemele, C.R., and Rundora, L. "Natural convection flow of heat generating hydro magnetic couple stress fluid with time periodic boundary conditions", Alex Eng. J., 57, pp. 1977-1989 (2018).

8. Adesanya, S.O., Souayeh, B., Gorji, M.R., et al. "Heat irreversibility analysis for a couple stress fluid flow in an inclined channel with isothermal boundaries", $J$. Taiwan Inst. Chem. E, 101, pp. 251-258 (2019).

9. Adesanya, S.O., Egere, A.C., and Lebelo, R.S. "Entropy generation analysis for a thin couple stress film flow over an inclined surface with Newtonian cooling", Physica A, 528, p. 121260 (2019).

10. Ashmawy, E.A. "Drag on a slip spherical moving in a couple stress fluid", Alex. Eng. J., 55, pp. 1159-1164 (2016). 
11. Ashmawy, E.A. "Effects of surface roughness on a couple stress fluid flow through corrugated tube", Eur. J. Mech. B Fluids., 76, pp. 365-374 (2019).

12. Adesanya, S.O., Egere, A.C., and Lebelo R.S. "Entropy generation analysis for a thin couple stress film flow over an inclined surface with Newtonian cooling", Physica A, 528, p. 121260 (2019).

13. Hassan, A.R. "The entropy generation analysis of a reactive hydromagnetic couple stress fluid flow through a saturated porous channel", Appl. Math. Comput., 369, p. 124843 (2020).

14. Zeeshan, A., Hussain, F., Ellahi, R., et al. "A study of gravitational and magnetic effects on coupled stress bi-phase liquid suspended with crystal and Hafnium particles down in steep channel", J. Mol. Liq., 286, pp. 110898-11908 (2019).

15. Ellahi, R. "The effects of MHD and temperature dependent viscosity on the flow of non-Newtonian nanofluid in a pipe: Analytical solutions", Appl. Math. Model, 37, pp. 1451-1467 (2013).

16. Jabeen, S., Hayat, T., Alsaedi, A., et al. "Consequences of activation energy and chemical reaction in radiative of tangent hyperbolic nanoliquid", Sci. Iran., 26, pp. 3928-3937 (2019).

17. Bayat, R. and Rahimi, A.B. "Numerical solution to $\mathrm{N}-\mathrm{S}$ equations in the case of unsteady axisymmetric stagnation-point flow on a vertical circular cylinder with mixed convection heat transfer", Sci. Iran., 25, pp. 2130-2143 (2018).

18. Ellahi, R. and Riaz, A. "Analytical solutions for MHD flow in a third grade fluid with variable viscosity", Math. Comput. Model, 52, p. 1783 (2010).

19. Hayat, T., Ellahi, R., and Asghar, S. "The influence of variable viscosity and viscous dissipation on the nonNewtonian flow: an analytical solution", Commun. Nonlinear Sci. Numer. Simul., 12, p. 300 (2007).

20. Nazeer, M., Ahmad, F., Saleem, A., et al. "Effects of constant and space-dependent viscosity on EyringPowell fluid in a pipe: Comparison of the perturbation and explicit finite difference methods", Z. Naturforsch, 74, pp. 961-969 (2019).

21. Ahmad, F., Nazeer, M., Saleem, A., et al. "Heat and mass transfer of temperature-dependent viscosity models in a pipe: Effects of thermal radiation and heat generation", Z. Naturforsch, 75, pp. 225-239 (2020).

22. Nazeer, M., Ali, N., Ahmad, F., et al. "Numerical and perturbation solutions of third-grade fluid in a porous channel: Boundary and thermal slip effects", Pramana-J. Phys., 94, p. 44 (2020).

23. Nazeer, M., Ali, N., Ahmad, F., et al. "Effects of radiative heat flux and joule heating on electroosmotically flow of non-Newtonian fluid: Analytical approach", International Communications in Heat and Mass Transfer, 117, p. 104744 (2020).

24. Nazeer, M., Ahmad, F., Saeed, M., et al. "Numerical solution for flow of a Eyring-Powell fluid in a pipe with prescribed surface temperature", J. Braz. Soc. Mech. Sci. Eng., 41, p. 518 (2019).
25. Ahmad, F., Tohidi, E., and Carrasco, J.A. "A parameterized multi-step Newton method for solving systems of nonlinear equations", Numer. Algorithms, 71, pp. 631-653 (2016).

26. Ali, N., Nazeer, M., Javed, T., et al. "Buoyancy driven cavity flow of a micropolar fluid with variably heated bottom wall", Heat Trans. Res., 49, pp. 457481 (2018).

27. Nazeer, M., Ali, N., and Javed, T. "Effects of moving wall on the flow of micropolar fluid inside a right angle triangular cavity", Int. J. Numer. Methods Heat Fluid Flow, 28, pp. 2404-2422 (2018).

28. Ali, N., Nazeer, M., Javed, T., et al. "A numerical study of micropolar flow inside a lid-driven triangular enclosure", Meccanica, 53, pp. 3279-3299 (2018).

29. Nazeer, M., Ali, N., and Javed, T. "Natural convection flow of micropolar fluid inside a porous square conduit: effects of magnetic field, heat generation/absorption, and thermal radiation", J. Porous Med., 21, 953-975 (2018).

30. Nazeer, M., Ali, N., Javed, T., et al. "Natural convection through spherical particles of a micropolar fluid enclosed in a trapezoidal porous container", Eur. Phys. J. Plus., 133, p. 423 (2018).

31. Hayat, T., Khan, M.I., Farooq, M., et al. "Impact of Cattaneo-Christov heat flux model in flow of variable thermal conductivity fluid over a variable thicked surface", I. Int. J. Heat Mass Transf., 99, pp. 702710 (2016).

32. Khan, M.I., Hayat, T., Qayyum, S., et al. "Entropy generation in radiative motion of tangent hyperbolic nanofluid in presence of activation energy and nonlinear mixed convection", Phys. Lett. A, 382, pp. $2017-$ 2026 (2018).

\section{Appendix}

List of constants used in the study:

$$
\begin{aligned}
& a_{0}=-\frac{G}{B_{0}^{4}} ; \quad a_{1}=\frac{G}{2 B_{0}^{2}} ; \quad a_{2}=\frac{G \operatorname{Sech}\left[B_{0}\right]}{B_{0}^{4}} ; \\
& a_{3}=\frac{5 G}{24} ; \quad a_{4}=-\frac{G}{4} ; \quad a_{5}=\frac{G}{24} ; \\
& a_{6}=-\frac{37 b^{2} G}{2520} ; \quad a_{7}=\frac{1}{45} B_{0}^{2} G ; \\
& a_{8}=-\frac{1}{120} B_{0}^{2} G ; \quad a_{9}=\frac{B_{0}^{2} G}{1260} ; \quad a_{10}=\frac{1}{24} G ; \\
& a_{11}=\frac{1}{2} S a_{10}\left(-1+A_{1} b_{18}\right) ; \quad a_{12}=\frac{1}{5} S a_{10} A_{1} b_{19} ; \\
& a_{13}=\frac{1}{30} S a_{10}\left(1+A_{1}\left(45 b_{17}-b_{18}\right)\right) ; \\
& a_{14}=-\frac{2}{105} S a_{10} A_{1} b_{19} ; \quad a_{15}=-\frac{5}{14} S a_{10} A_{1} b_{17}
\end{aligned}
$$




$$
\begin{aligned}
& a_{16}=\frac{2}{45} S a_{10} A_{1} b_{17} ; \quad a_{17}=-\frac{1}{330} S a_{10} A_{1} b_{17} \\
& a_{18}=\left(5 a_{11}+14 a_{13}+27 a_{15}+44 a_{16}+65 a_{17}\right) \text {; } \\
& a_{19}=\frac{1}{3}\left(7 a_{12}+18 a_{14}\right) ; \\
& a_{20}=\left(-6 a_{11}-15 a_{13}-28 a_{15}-45 a_{16}-66 a_{17}\right) \text {; } \\
& a_{21}=\left(\frac{1}{3}\left(-10 a_{12}-21 a_{14}\right)\right) ; \\
& b_{0}=\frac{1}{2}-\frac{7 G^{2} B_{r}^{\Theta}}{4 B_{0}^{8}}-\frac{G^{2} \operatorname{Sech}\left[B_{0}\right]^{2} B_{r}^{\Theta}}{8 B_{0}^{8}} \\
& +\frac{3 G^{2} \operatorname{Sech}\left[B_{0}\right]^{2} B_{r}^{\Theta}}{4 B_{0}^{6}} \\
& b_{1}=\frac{1}{2}-\frac{G B_{r}^{\Theta}}{6 B_{0}^{2}}+\frac{G B_{r}^{\Theta} \tanh \left[B_{0}\right]}{B_{0}^{5}} \text {; } \\
& b_{2}=\frac{G^{2} B_{r}^{\Theta}}{2 B_{0}^{6}}-\frac{3 G^{2} \operatorname{Sech}\left[B_{0}\right]^{2} B_{r}^{\Theta}}{4 B_{0}^{6}} ; \\
& b_{3}=-\frac{G B_{r}^{\Theta}}{6 B_{0}^{2}} ; \quad b_{4}=-\frac{2 G^{2} \operatorname{Sech}\left[B_{0}\right] B_{r}^{\Theta}}{B_{0}^{8}} ; \\
& b_{5}=-\frac{G^{2} \operatorname{Sech}\left[B_{0}\right]^{2} B_{r}^{\Theta}}{8 B_{0}^{8}} ; \quad b_{6}=-\frac{G \operatorname{Sech}\left[B_{0}\right] B_{r}^{\Theta}}{B_{0}^{5}} ; \\
& b_{7}=\frac{1}{2}+\frac{11 G^{2} B_{r}^{\Theta}}{120 B_{0}^{2}} ; \quad b_{8}=\frac{1}{2} ; \quad b_{9}=-\frac{G^{2} B_{r}^{\Theta}}{8 B_{0}^{2}} ; \\
& b_{10}=\frac{G^{2} B_{r}^{\Theta}}{24 B_{0}^{2}} ; \quad b_{11}=-\frac{G^{2} B_{r}^{\Theta}}{120 B_{0}^{2}} ; \\
& b_{12}=-\frac{1181 G^{2} B_{r}^{\Theta}}{453600} ; \quad b_{13}=\frac{1}{45} G^{2} B_{r}^{\Theta} ; \\
& b_{14}=-\frac{11}{400} G^{2} B_{r}^{\Theta} ; \quad b_{15}=\frac{11 G^{2} B_{r}^{\Theta}}{1260} ; \\
& b_{16}=-\frac{11 G^{2} B_{r}^{\Theta}}{12960} ; \quad b_{17}=-\frac{24 a_{10}^{2} B_{r}^{\Theta}}{5 B_{0}^{2}} \\
& b_{18}=\frac{1}{2}\left(1-22 b_{17}\right) ; \quad b_{19}=\frac{1}{2} ; \\
& b_{20}=24 a_{10} a_{20} ; \quad b_{21}=24 a_{10} a_{21} ; \\
& b_{22}=4 a_{10}\left(6 a_{11}-a_{20}+3 S a_{10}\left(-1+A_{1} b_{18}\right)\right) ; \\
& b_{23}=\frac{12}{5} a_{10}\left(10 a_{12}-3 a_{21}+3 S a_{10} A_{1} b_{19}\right) \text {; } \\
& b_{24}=\frac{8}{5} a_{10}\left(-6 a_{11}+15 a_{13}+S a_{10}(2\right. \\
& \left.\left.+A_{1}\left(45 b_{17}-2 b_{18}\right)\right)\right)
\end{aligned}
$$

$$
\begin{aligned}
b_{25}= & -\frac{8}{7} a_{10}\left(10 a_{12}-21 a_{14}+2 S a_{10} A_{1} b_{18}\right) ; \\
b_{26}= & -\frac{2}{7} a_{10}\left(45 a_{13}-84 a_{15}+S a_{10}(1\right. \\
& \left.\left.+A_{1}\left(135 b_{17}-b_{18}\right)\right)\right) ; \\
b_{27}= & \frac{2}{9} a_{10}\left(-63 a_{14}+S a_{10} A_{1} b_{19}\right) ; \\
b_{28}= & \frac{8}{15} a_{10}\left(-28 a_{15}+45 a_{16}+18 S a_{10} A_{1} b_{17}\right) ; \\
b_{29}= & -\frac{4}{33} a_{10}\left(135 a_{16}-198 a_{17}+11 S a_{10} A_{1} b_{17}\right) ; \\
b_{30}= & \frac{8}{91} a_{10}\left(-198 a_{17}+S a_{10} A_{1} b_{17}\right) ; \\
b_{31}= & -\left(b_{20}+b_{22}+b_{24}+b_{26}+b_{28}+b_{30}+b_{32}\right) ; \\
b_{32}= & -\left(b_{21}+b_{23}+b_{25}+b_{27}\right) ; \\
A_{1}= & \frac{x_{0}}{y_{0}^{2}} ; \quad S=e^{\left(\frac{x_{0}}{y_{0}}-\theta_{w}\right)} .
\end{aligned}
$$

\section{Biographies}

Fayyaz Ahmad obtained his doctorate from the Department of Mathematics in Computer Science and Computational Mathematics from the University of Insubria, Como, Italy, in December 2018 and is currently Assistant Professor in the Department of Applied Sciences at the National Textile University, Faisalabad. He is an expert in developing numerical algorithms. His areas of research include numerical analysis and fluid mechanics.

Mubbashar Nazeer obtained his doctorate from the Department of Mathematics and Statistics in Applied Mathematics (Fluid Mechanics) from the International Islamic University, Islamabad, in July 2018. He served as Assistant Professor in the Department of Mathematics at Riphah International University, Faisalabad Campus, for almost two years, and is currently Assistant Professor in the Department of Mathematics at the Institute of Arts and Sciences, Government College University Faisalabad, Chiniot Campus. His research interests include cavity flows, Newtonian and nonNewtonian fluids, analytical and numerical methods, bio-fluid mechanics, porous medium, heat and mass transfer analyses and computational fluid dynamics.

Waqas Ali obtained an MS degree from the University of Grenoble, France in Environmental Fluid Mechanics, and an MS degree in Mathematics from the International Islamic University, Islamabad, Pakistan. Dr. 
Waqas Ali is currently a researcher at the ThermoPlastic Composites Research Center (TPRC) and in the group of Production Technology (PT) at the University of Twente, Netherlands. Using analytical and computational mathematical techniques, his research work focuses on fluid flows related to various industrial applications such as composite materials, and largescale environmental flows.

Adila Saleem obtained her MS degree in Applied Mathematics (Fluid Mechanics) from the Department of Mathematics at Riphah International University, Faisalabad Campus, in January 2020, under the supervision of Dr. Nazeer. Her area of research is fluid mechanics.

Hafsa Sarwar obtained her MS degree in Applied Mathematics (Fluid Mechanics) from the Department of Mathematics at Riphah International University, Faisalabad Campus, in January 2020, under the supervision of Dr. Nazeer.

Samrah Suleman obtained her MS degree in Applied Mathematics (Fluid Mechanics) from the Department of Mathematics at Riphah International University, Faisalabad Campus, in January 2020, under the supervision of Dr. Nazeer.

Zahra Abdelmalek graduated in Applied Mathematics and currently working as a Professor at Duy Tan University, Vietnam. She has published various impact factor research articles in applied mathematics. Her areas of interest include viscous and non-Newtonian fluids, porous medium, nano fluid, hybrid nanofluid, heat and mass transfer analysis, and computational fluid dynamics. 